\title{
Espectro obsessivo-compulsivo: uma revisão
}

\section{Obsessive-Compulsive Spectrum: a revision}

\author{
Fernando Filipe Paulos Vieira*
}

\begin{abstract}
Resumo
A definição do espectro obsessivo-compulsivo indica um conjunto de transtornos que se caracterizam por pensamentos obsessivos (obsessões) e por comportamentos repetitivos ou atos mentais (compulsões). O desenvolvimento desses transtornos associa-se a uma base multifatorial que abrange fatores de ordem genética, neurológica, e psicológica. O presente artigo objetiva revisar alguns aspectos que englobam os principais aspectos sintomatológicos dos transtornos que integram o espectro obsessivo-compulsivo e as teorias explicativas que incluem os aspectos de ordem genética, neurológica e psicológica. Palavras-chave: Obsessão-Compulsão. Genética. Neurologia. Psicologia.
\end{abstract}

\begin{abstract}
The definition of the obsessive-compulsive spectrum indicates a set of disorders that are characterized by obsessive thoughts (obsessions) and by repetitive behaviors or mental acts (compulsions). The development of these disorders is associated with a multifactorial base that encompasses genetic, neurological, and psychological factors.This article aims to review some aspects that encompass the main symptomatological aspects of the obsessive-compulsive spectrum disorders, and the explanatory theories that include the genetic, neurological and psychological aspects.
\end{abstract}

Key-words: Obsession-Compulsion. Genetic. Neurology. Psychology.

* Psicólogo, graduado pela Universidade Lusófona do Porto e Mestre em Psicologia Clínica e da Saúde 


\section{Transtorno obsessivo-compulsivo e transtorno de acumulação}

\section{Caracterização Diagnóstica}

Ao contrário do que acontece com outros transtornos de ansiedade, em que o perigo se configura num objeto ou situação externos, no TOC - Transtorno Obsessivo-Compulsivo, o evento temeroso é um pensamento, imagem, ou impulso que o paciente tenta evitar por completo de igual forma que alguém com uma coulrofobia (fobia de Palhaços) tenta evitar o contato com palhaços (CLARK; O'CONNOR, 2005). Então, o TOC caracteriza-se pela presença de obsessões e/ou compulsões.

Por obsessões entendem-se pensamentos, imagens, ideias, ou impulsos intrusivos e recorrentes que são persistentes e incontroláveis (por exemplo, o indivíduo é incapaz de ignorar os pensamentos) e que são geralmente de caráter irracional aos olhos de quem as vivencia. Os tipos de obsessões mais comuns abrangem o medo de ser contaminados, os impulsos sexuais ou agressivos, os problemas corporais, e a ordem ou simetria (BLOCH; LANDEROS-WEISENBERGER; SEM; DOMBROWSKI KELMENDI; CORIC; LECKMAN, 2008). Os indivíduos com obsessões podem também estar sujeitos a dúvidas extremas, procrastinação, e indecisão.

Por sua vez, as compulsões são comportamentos ou atos mentais repetitivos, e claramente exacerbados que o indivíduo se sente compelido a concretizar para ver reduzida a ansiedade causada pelos pensamentos obsessivos ou para prevenir a ocorrência de alguma calamidade. Mesmo que o indivíduo detenha o entendimento racional de que não há necessidade para a execução do comportamento compulsivo, o indivíduo sente como se algo danoso poderá suceder a qualquer momento caso as compulsões não são concretizadas. De uma forma comum, as compulsões apresentadas englobam os seguintes tipos: Impulsos pela limpeza e ordenação, por vezes através de rituais elaborados; Concretização de atos mágicos de caráter protetor, tais como contar ou tocar numa 
determinada parte do corpo; Verificação repetitiva para ter a certeza de que certos atos foram realizados - por exemplo, retornar sete ou oito vezes a casa para averiguar se as luzes foram apagadas, as torneiras fechadas, ou as portas trancadas.

Com frequência se ouve falar de indivíduos que jogam, ingerem alimentos e bebidas alcoólicas compulsivamente. Mesmo que esses indivíduos possam apresentar um impulso irresistível para jogar, comer, ou beber, os clínicos não consideram tais comportamentos como compulsões pelo fato de tais comportamentos serem perpetrados na generalidade como experiência prazerosa. Com base num estudo, $78 \%$ dos indivíduos com compulsões viam os seus rituais como "um pouco estranhos ou absurdos" apesar das dificuldades em deixar de concretizá-los (STERN; COBB, 1978).

O TOC tende a ter início tanto antes dos 10 anos de idade quanto nos derradeiros anos da adolescência/começo da idade adulta. O transtorno tem sido descrito em crianças pequenas de aproximadamente 2 anos de idade (RAPOPORT, 1991). Entre os adultos, aproximadamente $1 \%$ preenchera os critérios de diagnóstico numa dada idade (JACOBI; WITTCHEN; HOLTING; HOFLER; PFISTER; MULLER; LIEB, 2004, RUSCIO; STEIN; CHIU; KESSLER, 2010; TORRES; PRINCE; BEBBINGTON; BHUGA; BRUGHA; FARRELL; SINGLETON, 2006), e cerca de $2 \%$ preencheram os critérios diagnósticos durante o tempo de vida (RUSCIO; STEIN; CHIU; KESSLER, 2010). O TOC é ligeiramente mais comum no sexo feminino (JACOBI et al., 2004; TORRES et al., 2006). O padrão da sintomatologia surge com similaridades entre culturas (SEEDAT; MATSUNAGA, 2006). O TOC é uma psicopatologia crônica - um estudo com base num follow-up de 40 anos de indivíduos hospitalizados com diagnóstico de TOC nos anos 1950 mostrou que somente 20\% apresentou recuperação completa (SKOOG; SKOOG, 1999). Mais do que três quartos dos indivíduos com TOC apresentam comorbidades com outros transtornos de ansiedade durante o tempo de vida, e cerca dois terços apresentam comorbidades com o transtorno depressivo maior durante ao longo da vida. $\mathrm{O}$ abuso de substâncias é também comum entre indivíduos portadores de TOC (RUSCIO et al., 2010). Aproximadamente um terçO dos indivíduos com TOC experimentam, ao menos alguns sintomas de transtorno de acumulação (STEKETEE; FROST, 2003). 
Em relação ao fenômeno da acumulação, colecionar é o hobby favorito de muitos indivíduos. Mas, o que distingue um simples fascínio por coleções do transtorno clínico de acumulação? Para o indivíduo com transtorno de acumulação, a necessidade de adquirir é somente parte do problema. O problema maior reside no fato dele odiar tomar partido dos seus objetos, mesmo quando deixam de ter qualquer valor. De um modo típico, o indivíduo adquiriu uma grande variedade de objetos - coleções de roupas, telas, ou antiguidades podem ser adicionadas a velhos contêineres ou rolhas de garrafas. Cerca de dois terços dos indivíduos que acumulam objetos parece não possuir o conhecimento acerca da severidade dos seus atos (STEKETEE; FROST, 2003). Os indivíduos com transtorno de acumulação são extremamente ligados às suas possessões, e apresentam resistência aos esforços para se desfazerem dos seus objetos.

Aproximadamente um terço dos indivíduos com transtorno de acumulação, com maior frequência do sexo feminino, acumulam animais (PATRONEK; NATHANSON, 2009). Os indivíduos que engajam na acumulação de animais vêem-se, por vezes, como salvadores de animais, porém aqueles que testemunham o problema vêem de forma diferente - o número de animais acumulados melhora a capacidade individual para proporcionar um tratamento adequado, abrigo, e comida.

As consequências da acumulação podem ser severas. Estudo de Kim, Steketee e Frost (2001) abordou cuidadores de idosos para descreverem os seus clientes que sofriam com o transtorno de acumulação e esses relataram que entre os seus clientes que tinham problemas com a acumulação havia extrema imundice habitacional em aproximadamente um terço dos indivíduos, caracterizada por odores insuportáveis desde comida podre ou fezes. Também, mais de $40 \%$ dos indivíduos acumularam tantos itens que se tornaram incapazes de usar a geladeira, a pia da cozinha, a banheira, e cerca de $10 \%$ dos indivíduos tornaram-se incapazes de usar o banheiro. As condições respiratórias, higiene empobrecida, e dificuldades em cozinhar podem contribuir para o enfraquecimento da saúde física. Muitos membros familiares são portadores de relacionamentos severos, e incapacidade em compreender o apego aos objetos. Cerca de três quartos dos indivíduos com transtorno de acumulação, segundo 
Frost et.al. (2009) engajam-se em compras excessivas e muitos se tornam incapazes de trabalhar, conforme haviam afirmado Tolim, Frost, Steketee, Gray e Fitch (2008); além disso, a pobreza se configure numa condição entre os indivíduos com esta condição , conforme afirmaram Samuels et al. (2007). Tendo em consideração a escalada do problema, os agentes de saúde estão geralmente envolvidos na tentativa de salvação para o problema. Aproximadamente $10 \%$ dos indivíduos diagnosticados com transtorno de acumulação apresentarão sentimento de medo em relação a serem despejados num dado momento das suas vidas (TOLIN et al., 2008). Para alguns indivíduos, o dinheiro gasto na aquisição de objetos conduz ao desamparo. Quando os animais estão envolvidos, as agências de proteção animal tornam-se envolvidas.

Nenhum estudo usou entrevistas diagnósticas estruturadas em amostras representativas com intuito de estimar a prevalência do transtorno de acumulação. Com base em escalas de auto-relatos, aproximadamente $2 \%$ da população detinha algum conhecimento no que diz respeito a problemas com os sintomas de acumulação (IERVOLINO, RIJSDIJK; CHERKAS; FULLANA; MATAIX-COLS, 2011). Apesar do fenômeno da acumulação ser mais comum entre homens do que em mulheres (SAMUELS et al., 2007), um escasso número de homens buscam por tratamento (STEKETEE; FROST, 2003). O comportamento de acumular começa geralmente na infância ou início da adolescência (GRISHAM; FROST; STEKETEE; KIM; HOOD, 2006). A sintomatologia precoce pode ser controlada pelos progenitores e através de ganhos reduzidos, os severos prejuízos provenientes da acumulação não é superficial até ao término da vida. A acumulação de animais, na sua generalidade não emerge até à meia idade ou idade mais avançada (PATRONEK; NATHANSON, 2009).

O transtorno de acumulação não é reconhecido como patologia até ao lançamento do DSM-5. Existem alguns debates sobre se o transtorno de acumulação deveria ser incluído no capítulo do transtorno obsessivo-compulsivo e transtornos relacionados ou se deveria ser incluído num apêndice para futuramente ser objeto de pesquisa. No DSM-IV-TR, o transtorno de acumulação é descrito como um sintoma que pode acompanhar o TOC. Embora o comportamento da acumulação seja comum no TOC, ele poderá ocorrer entre os 
indivíduos que não são portadores de sintomatologia obsessivo-compulsiva (BLOCH et al., 2008). A depressão, o TAG ${ }^{1}$, e a fobia social são comuns entre os indivíduos diagnosticados com transtorno de acumulação (MATAIX-COLS; FROST; PERTUSA; CLARK; SAXENA; LECKMAN WILHELM, 2010). Em algumas ocasiões, o transtorno de acumulação tem o seu desenvolvimento em indivíduos com esquizofrenia ou demência (HWANG; TSAI; YANG; LIU; LIRNG, 1998).

\section{A Etiologia}

\section{Teorias Genéticas}

Estudos envolvendo gêmeos têm evidenciado que se um gêmeo monozigótico (idêntico) é portador de TOC, o outro apresenta uma probabilidade de aproximadamente $65 \%$ de ter o transtorno. Como expectativa, caso esta elevada taxa reflita em genes comuns, a taxa é reduzida (apenas 15\%) em gêmeos dizigóticos (PAULS; RAYMOND; ROBERTSON, 1991). Além disso, como esperado a partir dos resultados vindos dos estudos com gêmeos, o TOC é mais comum entre os parentes dos indivíduos portadores do transtorno $(10.3 \%$ dos quais também possuem TOC) do que entre os parentes dos participantes do grupo de controle (dos quais apenas $2 \%$ tinham TOC) (PAULS; ALSOBROCK; GOODMAN; RASMUSSEN; LECKMAN, 1995).

Todavia, apesar dos estudos com famílias terem dado uma contribuição genética concernente ao TOC, a ligação não é nem simples nem direta: Os membros da família de um indivíduo com TOC detêm maior predisposição quando comparados com outros indivíduos a padecerem de um transtorno de ansiedade, mas que não tem que ser necessariamente o TOC (BLACK; NOYES; GOLDSTEIN; BLUM, 1992 , SMOLLER; FINN; WHITE, 2000; TORGERSEN, 1983).

Assim, é possível dizer que quando os pacientes aprendem acerca do TOC, eles reconhecem as condutas que eles mesmos perpetraram. Caso um indivíduo tenha essa reação quando há aprendizagem sobre os aspectos do TOC, ele deve se preocupar: o TOC pode ser um reflexo de um funcionamento extremo dos sistemas cerebrais que funcionam da mesma maneira em cada um do ser humano de modo

\footnotetext{
Transtorno de Ansiedade Generalizada.
} 
a produzirem brandas formas de pensamento. De acordo com este ponto de vista, o TOC não é um transtorno qualitativamente distinto, com sistemas do cérebro que produzem tipos anormais de resposta (a forma como as alucinações podem resultar em alguns transtornos psicóticos), mas raramente é somente um término de um continuum - com ancoragem "normal" na outra extremidade. Em suma, os pesquisadores (PAULS et al., 1995) constataram que os parentes dos indivíduos com TOC estavam mais predispostos ao TOC quanto a sintomatologia típica do TOC se configurava em aspectos sub-clínicos (não severamente suficientes para qualificar como transtorno) quando comparados com os indivíduos de grupo de controle. No que concerne aos sintomas sub-clínicos, o cérebro produz o mesmo tipo de pensamentos, impulsos, e imagens como acontece no TOC, mas não suficiente para prejudicar a qualidade de vida individual.

Em relação ao transtorno de acumulação, tendo em conta que é um fenótipo recente e distinto, estudos genéticos que são específicos ao transtorno de acumulação apenas se separaram do TOC quando passados 5 a 10 anos, implicando regiões relativas aos cromossomas 4q, 5q, 6q, 14q, 17q (ZHANG; LECKMAN; PAULS, 2002, SAMUELS; BIENVENU; PINTO, 2007, SAMUELS; SHUGART; GRADOS, 2007). Um número reduzido de genes previamente implicados no TOC, incluíndo aCOMT ${ }^{2}$, o NTRK33 $3^{3}$ o $B D N F^{4}$, e o $S L C 1 A 1^{5}$, mostraram alguns resultados positivos no transtorno de acumulação (LOCHNER; KINNEAR; HEMMINGS, 2005, ALONSO; GRATACOS; MENCHON, 2008, MUINOS-GIMENO; GUIDI; KAGERBAUER, 2009, WENDLAND; MOYA; TIMPANO, 2009). Porém, nenhum gene tem sido identificado de forma específica como gene responsável ao desenvolvimento do transtorno de acumulação (HIRSCHTRIT; MATHEWS, 2014).

\section{Teorias Neurológicas}

Quando o lobo frontal precipita uma ação, ele interage com os

\footnotetext{
2 Catecol O-Metiltransferase.

3 Recetor tipo 3 da Tirosina Neurotrópica Quinase.

4 Fator Neurotrópico Derivado do Cérebro.

5 Transportador de aminoácido excitatório.
} 
gânglios da base - e, por vezes essa interação enverga numa rede de atividades repetitivas (BREITER, RAUCH, KWONG, BAKER, WEISSKOFF; KENNEDY, 1996, JENIKE, 1984, RAUCH; JENIKE; ALPERT; BAER; BREITE; SAVAGE, 1994, RAUCH; DOUGHERTY; COSGROVE; CASSEM; ALPERT; PRICE, NIERENBERG; MAYBERG; BAER, JENIKE; FISHMAN, 2001). Um elevado número de pesquisadores tem acreditado que essa rede neuronal desempenha um papel crucial no desenvolvimento dos pensamentos obsessivos, que invadem a mente e são de difícil interrupção. A execução de uma compulsão pode interromper temporariamente os pensamentos obsessivos através da redução da atividade neuronal (INSEL, 1992; JENIKE, 1984; MODELL, MOUNTZ, CURTIS, GREDEN, 1989). (Mas logo depois que o comportamento compulsivo termina, há um retorno das obsessões).

Consistente com estas ideias, Rapoport (1991) sugeriu que os sintomas do TOC poderiam ser causados por conexões disfuncionais entre o lobo frontal, o tálamo, e os gânglios da base. Muitos pesquisadores têm-se focado nas possíveis anormalidades existentes nos gânglios da base e no lobo frontal em particular (INSEL, 1992; PIGOTT, MYERS, WILLIAMS, 1996; SAXENA, RAUCH, 2000). Na verdade, como previsto pela teoria, tanto o córtex frontal (em especial o córtex órbitofrontal ${ }^{6}$ - a parte menor do córtex, situada atrás dos olhos) e os gânglios da base funcionam de um modo deficiente em indivíduos com TOC (BAXTER, 1992; BERTHIER; KULISEVSKY; GIRONELL; LÓPEZ, 2001, SAXENA; BRODY; SCHWARTZ; BAXTER, 1998). Essa deficiência no funcionamento poderia prevenir de modo eficiente o lobo frontal na respectiva ação de interromper a rede de atividades neuronais repetitivas, como acontece em indivíduos sem TOC.

Pesquisadores têm também sugerido a existência de uma associação entre o TOC e um excesso de matéria cinzenta no lobo frontal e escassa quantidade da mesma matéria nas porções posteriores do cérebro (KIM; LEE; KIM; KIM; KIM; HAN, 2001). Tais anormalidades estruturais são consistentes com o invulgar aumento da atividade

6 Região do córtex pré-frontal envolvida no processamento cognitivo da tomada de decisão. 
na região frontal dos indivíduos portadores de TOC quando a visualização de um estímulo que provoca os pensamentos e comportamentos relacionados ao TOC (ADLER, MCDONOUGH-RYAN; SAX; HOLLAND; ARNDT; STRAKOWSKI, 2000). Além disso, esses indivíduos apresentam deficiências nas habilidades espaço-visuais, que confiam a áreas similares do cérebro (MICALLEF; BLIN, 2001).

Contudo, nem todos os estudos encontraram tais deficiências cerebrais (BAXTER; SCHWARZ; GUZE, 1991), que possam sugerir que o TOC pode resultar de mais de uma maneira (HOLLANDER; LIEBOWITZ; ROSEN, 1991). Na verdade, os pesquisadores constataram que muitos (mas não todos) os indivíduos diagnosticados com TOC tiveram complicações ao nascerem, epilepsia, trauma cerebral, ou infecção do cérebro ou das membranas que o cobrem. Por exemplo, quando as crianças desenvolvem rapidamente a sintomatologia do TOC, a causa pode ser um tipo particular de infeção estreptocócica que, por seu turno leva a um transtorno no sistema imunológico, que é conhecido por transtorno neuropsiquiátrico autoimune associado à infecção estreptocócica ${ }^{7}$. Esse transtorno afeta os gânglios da base (SWEDO; LEONARD; GARVEY; MITTLEMAN; ALLEN; PERLMUTTER, 1998). A sintomatologia do TOC desaparece quando às crianças são administrados os antibióticos apropriados.

No caso do transtorno de acumulação, tem havido algumas descobertas recentes com base em estudos por neuroimagem que reforçam a necessidade para uma distinção entre o transtorno de acumulação e o TOC. Estudos recentes revelam que a disfunção no córtex cingulado anterior ${ }^{8}$ pode mediar tanto os sintomas quanto os déficits neurocognitivos associados à compulsão por acumulação (SLYNE; TOLIN, 2014). As regiões - frontal e temporal podem modular ou suprimir subcorticalmente as predisposições dirigidas à aquisição e salvação e, portanto a inibição ou o dano nessas áreas pode resultar numa coleção orgânica de comportamentos acumulativos.

Além disso, a atividade neuronal no transtorno de acumulação parece ter diferenças em relação ao TOC. Por exemplo, no transtorno

Transtornos causados por varias espécies de Streptococcus.

8 Parte frontal do córtex cingulado cujo formato é semelhante ao de um "colar" que cerca a parte frontal do corpo caloso. 
de acumulação, a hipoatividade no estado de repouso pode ajudar na explicação do baixo insight e motivação, e a hiperatividade quando a necessidade de tomar decisões pode ajudar a explicar a subjetiva indecisão, apego excessivo, e afeto negativo durante o ato de descartar os objetos (SAXENA; RAUCH, 2000). Em linha com essas diferenças neuro-anatômicas relativas ao transtorno de acumulação, alguns estudos têm mostrado que os medicamentos que aumentam a atividade do córtex cingulado anterior podem conduzir a algumas alterações no comportamento de acumulação. Esses medicamentos incluem os estimulantes (DE HAES; MEGUIRE; JAGER, 2007), o modafinil (SPENCE, GREEN; WILKONSON, 2005, JOO; TAE; JUNG, 2008), os inibidores de colinesterase (NAHAS;GEORGE; HORNER, 2003, DE BRUIJN; HULSTIJN; VERKES, 2004), ou a memantina (CHOW; FAM; GRAFF-GUERRERO, 2012). Além da hipoatividade notada por Saxena e Rauch (2000), foi também notada a existência de um sintoma provocador da hiperatividade, conforme Mataix-Cols, Wooderson e Lawrence (2004) e também anunciado por Tolin, Kiehl, e Worhunsky (2009). Essa realidade parece demonstrar uma deficiência bifásica no funcionamento do córtex cingulado anterior e do libo da ínsula em indivíduos com diagnóstico de transtorno de acumulação. As regiões do lobo da ínsula do córtex cingulado anterior sugerem anormalidades na identificação da significância emocional em relação a um estímulo, geração de uma resposta emocional apropriada, ou regulação dos estados afetivos durante a execução da tomada de decisão. Então, os estudos por neuroimagem referentes ao transtorno de acumulação implicam uma gama de regiões cerebrais frontais e temporais, que podem elevar ou diminuir as movimentações corticais e subcorticais no transtorno de acumulação. Existe claramente uma necessidade para maiores pesquisas nesta área e uma distinção mais cuidadosa entre o TOC e o transtorno de acumulação.

\section{Teorias Psicológicas}

Do ponto de vista da aprendizagem, o conceito psicológico do condicionamento tem sido usado para compreender o desenvolvimento das obsessões e das compulsões (BAER; MINICHIELLO, 1990). As compulsões, enquanto mentais ou observáveis, diminuem 
geralmente a ansiedade engendrada pelos pensamentos obsessivos. Por isso, se um indivíduo se encontra preocupado com o medo de ser contaminado por germes, a repetitiva lavagem das mãos diminui a ansiedade causada por tais medos. A compulsão torna-se uma resposta condicionada à ansiedade. Devido ao aspecto redutor de tensão da compulsão, este comportamento aprendido torna-se reforçado e eventualmente estabelecido (RACHMAN; HODGSON, 1980). As compulsões, pelo contrário, reforçam realmente a ansiedade porque elas previnem a ocorrência da habituação; ou seja, a partir do desempenho de uma compulsão, o contato com o estímulo que evoca o medo (por exemplo, a sujeira) não é mantido, e o habito (diminuição do medo associado ao estímulo) deixa de ocorrer. Este modelo teórico da aprendizagem do TOC tem tido uma influência maior no modo como a terapia comportamental é usada no tratamento do transtorno.

No que concerne ao transtorno de acumulação, a mesma resposta à ansiedade evocada pelas obsessões que leva às compulsões não é o motivador central dos sintomas. Assim, o transtorno de acumulação parece ser egosistônico ao invés de egodistônico como acontece no TOC (RACHMAN; ELLIOT; SHAFRAN, 2009). O sintoma central do transtorno de acumulação envolve a acumulação de itens que desencadeia uma desordem substancial que pode interferir com o uso funcional do espaço de vida individual e que pode resultar em condições insalubres ou risco de incêndio, representando um perigo à saúde pública. $\mathrm{O}$ estresse significativo é maior desencadeador da sintomatologia, evocado pela incapacidade individual de viver isolado das suas possessões (PERTUSA; FROST; FULLANA, 2010, WHEATON; ABRAMOVITZ; FABRICANT, 2011).

Do ponto de vista da psicanálise, a mesma baseia-se no conceito de que a sintomatologia psiquiátrica possui significado que deriva dos conflitos inconscientes. Os conflitos inconscientes entre a passividade e a agressão, ou entre a obediência e o desafio, podem levar à ansiedade. A ansiedade produzida por tais conflitos conduz à formação das obsessões e compulsões bem como das defesas de formação reativa, isolamento (FREUD, [1917-1919] 1963; MACKINNON; MICHELS, 1971). 


\section{Transtorno dismórfico corporal}

\section{Caracterização Diagnóstica}

Os indivíduos com transtorno dismórfico corporal ou dismorfofobia, demonstram preocupação referente a um defeito imaginário ou exacerbado nas suas aparências. Embora os indivíduos com transtorno dismórfico corporal possam parecer atrativos aos olhos dos outros, eles percebem-se como feios ou mesmo "monstros" (PHILLIPS, 2006). As mulheres tendem a focarem-se na pele, lábios, seios, e pernas, ao passo que os homens são mais suscetíveis para focarem-se na altura, tamanho do órgão genital, ou cabelos do corpo (PERUGI; AKISTAL; GIANNOTTI; FRARE; DI VAIO; CASSANO, 1997). Alguns indivíduos do sexo masculino sofrem por se preocuparem com a ideia de que os seus corpos são pequenos ou pouco musculosos, mesmo quando outros indivíduos não se deparem com essa percepção.

À semelhança dos indivíduos com TOC, os indivíduos com transtorno dismórfico corporal encontram dificuldades no que diz respeito à interrupção dos pensamentos acerca dos seus conceitos. Em média, os indivíduos com transtorno dismórfico corporal pensam acerca da respectiva aparência durante um período de 3 a 8 horas diárias (PHILLIPS; WILHELM; KORAN; DIDIE; FALLON; FEUSNER; STEIN, 2010). Ainda à semelhança dos indivíduos com TOC, os indivíduos com transtorno dismórfico corporal sentem-se compelidos a engajar em determinados comportamentos. No transtorno dismórfico corporal, os comportamentos compulsivos mais comuns incluem a averiguação da aparência diante do espelho, comparação da aparência com a de outras pessoas, procura da opinião de outras pessoas como comprovativo da sua opinião no âmbito da aparência, ou uso de estratégias para alterar a aparência ou camuflagem das áreas corporais desgostosas (PHILLIPS et al., 2010). Enquanto muitos indivíduos despendem horas diárias verificando a respectiva aparência, alguns tentam evitar serem lembrados pelas falhas percebidas através do comportamento de evitar colocar-se diante do espelho, de superfícies reflexivas, ou de luzes brilhantes (ALBERTINI; PHILLIPS, 1999).

Os sintomas do transtorno dismórfico corporal são extrema- 
mente estressantes. Cerca de um terço dos indivíduos portadores do transtorno descrevem delírios referentes à aparência, tais como convencimento de que os outros se riem deles ou olham para as suas falhas (PHILLIPS, 2006), e aproximadamente um quarto dos portadores de transtorno dismórfico corporal se submetem a intervenções de cirurgia plástica (PHILLIPS; MCELROY; DWIGHT; EISEN; RASMUSSEN, 2001). Infelizmente, a cirurgia plástica é insuficiente para aliviar os conceitos dos pacientes de transtorno dismórfico corporal (VEALE, 2000), e muitos deles demonstram vontade de processar ou ferir os próprios cirurgiões logo após a cirurgia, pelo fato de se sentirem desapontados com o resultado. Aproximadamente um quinto dos indivíduos com o transtorno já pensou em cometer suicídio (RIEF; BUHLMANN; WILHELM; BORKENHAGEN; BRAHLER, 2006).

A preocupação com a aparência pode interferir em muitos aspectos do funcionamento ocupacional e social. Os indivíduos diagnosticados com transtorno dismórfico corporal experimentam frequentemente níveis elevados de vergonha, ansiedade, e depressão com relação à aparência, e são comuns várias respostas comportamentais a esses sentimentos poderosos. Alguns indivíduos podem evitar o contato com os outros pelo fato de temerem que os outros façam uma avaliação acerca da aparência, e por vezes esse medo são tão exacerbados que o indivíduo se torna incapaz de sair de casa. Aproximadamente $40 \%$ dos indivíduos com transtorno dismórfico corporal apresentam incapacidade para trabalhar (DIDIE; MENARD; STERN; PHILLIPS, 2008).

O transtorno dismórfico corporal ocorre de modo mais frequente no sexo feminino do que no sexo masculino, embora mesmo entre as mulheres a prevalência seja relativamente rara, menos de $2 \%$ (RIEF et al., 2006). Entre as mulheres que procuram cirurgias plásticas, todavia, cerca de 5 a $7 \%$ preenchem os critérios de diagnóstico de transtorno dismórfico corporal (ALTAMURA; PALUELLO; MUNDO; MEDDA; MANNU, 2001). De um modo típico, o transtorno dismórfico corporal tem início nos finais da adolescência. Por volta de $90 \%$ dos indivíduos diagnosticados com transtorno dismórfico corporal apresentam, após 1 ano da execução do diagnóstico sintomatologia do transtorno (PHILLIPS, 2006), mas acima de um período de 8 
anos, aproximadamente três quartos dos indivíduos com transtorno dismórfico corporal se recuperarão da sintomatologia (BJORNSSOM; DYCK; MOITRA; STOUT; WEISBERG; KELLER; PHILLIPS, 2011).

Os fatores sociais e culturais desempenham um sério papel no modo como os indivíduos decidem no que respeita à atratividade. Em relação aos estudantes, as preocupações acerca da aparência do corpo parecem ser mais comuns na América do que na Europa - aproximadamente $74 \%$ dos estudantes Americanos relatam, pelo menos alguma preocupação acerca da imagem corporal, com as mulheres a serem mais predispostas do que os homens no que diz respeito à insatisfação da aparência corporal (BOHNE; KEUTHERN; WILHELM; DECKERSBACK; JENIKE, 2002). A maior parte das preocupações, contudo, não são suficientemente extremas para serem caracterizadas como desordens psíquicas. Os indivíduos com transtorno dismórfico corporal experimentam estresse agonizado com relação à percepção dos seus defeitos físicos.

Os casos apresentados ao redor do mundo sugerem que os sintomas e os resultados do transtorno dismórfico corporal são similares entre culturas (PHILLIPS, 2005). A parte do corpo torna-se um foco de preocupação que, por vezes difere de cultura. Por exemplo, as preocupações relacionadas às pálpebras são mais comuns no Japão do que nos países Ocidentais. Os indivíduos Japoneses portadores de transtorno dismórfico corporal parecem demonstrar maior preocupação acerca da eventualidade de perpetrar uma ofensa aos outros comparativamente com os indivíduos Ocidentais (SUZUKI; TAKEI; KAWAI; MINABE; MORI, 2003).

Poucos indivíduos diagnosticados com transtorno dismórfico corporal preenchem os critérios de diagnóstico para outro transtorno. A comorbidade mais comum encontra-se em transtornos como transtorno depressivo, fobia social, TOC, transtornos por abuso de substâncias, e transtornos de personalidade (GUSTAD; PHILLIPS, 2003). Cuidados devem ser tomados no ato da distinção entre o transtorno dismórfico corporal e os transtornos do comportamento alimentar. A maioria dos indivíduos com transtorno dismórfico corporal preocupa-se com vários aspectos referentes à aparência. Quando o foco das preocupações são apenas a altura e o peso, os clínicos devem considerar se a sintomatologia é melhor explicada 
por um transtorno do comportamento alimentar.

\section{Etiologia}

\section{Teorias Genéticas}

O transtorno dismórfico corporal é mais comum em parentes de primeiro grau que possuam um diagnóstico de TOC, quando comparado com os indivíduos do grupo de controle, o que sugere uma etiologia partilhada entre os dois transtornos (BIENVENU; SAMUELS; WUYEK, 2011). Estudos em gêmeos apontam, de um modo semelhante, para a existência de uma predisposição genética entre o transtorno dismórfico corporal e o TOC, embora esses estudos tenham examinado o conceito amplo de "preocupação dismórfica" ao invés do transtorno dismórfico corporal (MONZANI; RIJSDIJK; IERVOLINO, 2012).

\section{Teorias Neurológicas}

O transtorno dismórfico corporal está associado ao processamento visual anormal, que consiste em um desvio no âmbito da codificação e análise detalhada das faces e objetos sem rosto, ao invés do uso holístico de estratégias do processamento visual (por exemplo, visualização de uma "grande imagem") (FEUSNER; MOODY; HEMBACHER, 2010, FEUSNER; HEMBACHER; MOLLER, 2011). Esta descoberta é consistente com as observações clínicas de que os indivíduos com transtorno dismórfico corporal se focam de um modo exacerbado em detalhes minúsculos da aparência enquanto ignoram os aspectos globais de como eles parecem. Dados preliminares sugerem também anormalidades no funcionamento executivo (DUNAI; LABUSCHANGE; CASTLE, 2010).

Um estudo com base em ressonância magnética constatou ausência de diferenças volumétricas entre os indivíduos com transtorno dismórfico corporal e os indivíduos do grupo de controle, ao passo que a partir de dois estudos se descobriu um volume excessivo de matéria branca nos indivíduos com diagnóstico de transtorno dismórfico corporal (PHILLIPS; WILHELM; KORAN, 2010). Um desses estudos se deparou também com um deslocamento para a esquerda na assimetria caudada, e o outro estudo descobriu que o córtex orbitofrontal e o cingulado anterior detinham tamanhos pequenos e 
que o tálamo era detentor de um tamanho excessivo (PHILLIPS et al., 2010). Estudos de pequena escala como o de Buschanan, Rossell e Maller (2013); e estudo de Feusner, Arienzo e Li (2013) sugerem que o transtorno dismórfico corporal pode ser caracterizado pelo fato de que as fibras da matéria branca se encontram comprometidas, ou seja, as conexões insuficientes, havedo empobrecimento da integração da informação entre as diferentes áreas do cérebro, em associação ao insight empobrecido existente em indivíduos com transtorno dismórfico corporal. Estudo de Feusner et al. (2013) constatou relativa hiperatividade localizada no córtex orbito frontal esquerdo quando os indivíduos visualizaram as suas próprias faces, refletindo de um modo possível na preocupação obsessiva enquanto os mesmos indivíduos visualizavam os seus próprios rostos. Este padrão de ativação é também uma característica do TOC.

\section{Teorias Psicológicas}

Os indivíduos com transtorno dismórfico corporal parecem demonstrar dificuldades no âmbito da identificação das expressões emocionais e, possuem uma tendência para a interpretação neutra dos rostos e cenátios como sendo ameaçadores (BUHLMANN; ETCOFF; WILHELM, 2006, PHILLIPS, 2009). Estas descobertas são consistentes com a frequente ocorrência de ideias/delírios de referência presentes no transtorno dismórfico corporal.

\section{Dermatotilexomania}

\section{Caracterização Diagnóstica}

A dermatotilexomania, também conhecida por transtorno de escoriação é um diagnóstico inédito cuja característica principal é a recorrente escoriação da própria pele. As regiões mais sofríveis envolvem a face, os braços, e as mãos, mas os indivíduos com o transtorno podem escoriar, espremer, ou esfregar uma gama de áreas, buscando pequenas irregularidades da pele, ou crostas. Alguns indivíduos com dermatotilexomania descrevem um impulso para picar, que é aliviado pela escoriação, enquanto que outros indivíduos se sentem compelidos a escoriar a pele de moto automático, sem plena consciência. Todos os indivíduos com dermatotilexomania 
relatam tentativas de diminuir ou interromper o comportamento de escoriação (LOCHNER; GRANT; ODLAUG, 2012), apesar de alguns deles evidenciarem um sentimento desesperançoso em relação ao livramento da escoriação.

O DSM-5 (American Psychiatric Association, 2013), requer que a dermatotilexomania cause estresse clinicamente significativo ou prejuízo no funcionamento social, ocupacional, ou em outras áreas do funcionamento individual. Os indivíduos podem sentir vergonha extrema, embaraço, ou perda do controle a respeito da incapacidade para interromper o comportamento de escoriação. Eles podem selecionar áreas particulares (por exemplo, a pele situada por cima ou por baixo dos braços) até que as lesões não serão propensas a serem vistas pelos outros; podem gastar uma considerada quantidade de tempo camuflando as lesões da pele nas áreas mais visíveis; e evitar situações sociais devido ao embaraço que sentem no âmbito das lesões (TUCKER; WOODS; FLESSNER, 2011).

A dermatotilexomania não pode ser atribuída a outra condição médica e não deve ser melhor explicada por outro transtorno psiquiátrico. Várias condições dermatológicas são caracterizadas por coceira (por exemplo, sarna). A dermatotilexomania pode ser precipitada pelos psicoestimulantes (por exemplo, cocaína), e pode ser vista como sintomatologia de várias condições psiquiátricas; por exemplo, os indivíduos com dermatotilexomania podem escoriar a pele como resposta a uma ideia delirante (por exemplo, parasitose) ou alucinações táteis (por exemplo, formigamento).

\section{Etiologia}

\section{Teorias Genéticas}

Estudo com gêmeos e famílias indica que a dermatotilexomania possui um importante componente genético, e é mais comum em parentes de indivíduos com TOC (MONZANI; RIJSDIJK; CHERKAS, 2012). Apesar da escassez de estudos no âmbito dos genes candidatos à dermatotilexomania, há uma evidência preliminar de uma associação entre a dermatotilexomania e os variantes no gene SAPAP3, que codifica uma série de proteínas que são intensamento expressados no estriado (GRANT; ODLAUG; CHAMBERLAIN, 2011). 


\section{Teorias Neurológicas}

Alguns indivíduos com dermatotilexomania podem demonstrar problemas na inibição motora, apontando para um envolvimento do circuito corticostriatal (GRANT et al., 2011). Com base numa perspectiva evolucionária, a dermatotilexomania pode ser conceituada em termos de preparação desordenada, que pode estabelecer alterações em importantes estruturas corticostriatais. Pesquisas preliminares de neuroimagem indicam uma anisotropia fraccional reduzida nos tratos da matéria branca associada à geração e supressão motora, proporcionando algum suporte hipotético. Estudos clínicos envolvendo animais podem, em última instância, dar luz aos circuitos dos sistemas moleculares que são mais relevantes para a dermatotilexomania (FEUSNER; HEMBACHER; PHILLIPS, 2009, GARNER; THOGERSON; DUFOUR, 2011).

\section{Teorias Psicológicas}

Os modelos cognitivo-comportamentais sugerem que a dermatotilexomania pode servir para modular os níveis de excitação. Tanto o reforço positivo (por exemplo, o prazer proporcionado pela escoriação) quanto o reforço negativo (por exemplo, a redução da ansiedade) têm sido hipotetizados como formas de manutenção da dermatotilexomania.

\section{Tricotilomania}

\section{Caracterização Diagnóstica}

A tricotilomania é caracterizada por um impulso recorrente de arrancar os próprios cabelos. As regiões pelosas incluem comumente o couro cabeludo, as sobrancelhas, ou as pálpebras, mas também as axilas, as áreas faciais, ou púbicas, ou outra área do corpo onde cresçam cabelos. Alguns indivíduos descrevem um impulso para puxar, que é aliviado através do comportamento de arrancar os cabelos, enquanto que outros se deparam com o fato de que o comportamento de puxar os cabelos consiste num ato automático, com relativa consciência de tal comportamento - por exemplo, enquanto o indivíduo dirige ou estuda. Todos os indivíduos com tricotilomania 
relatam tentativas de diminuir ou interromper o comportamento de arrancar os cabelos (LOCHNER; GRANT; ODLAUG, 2012), apesar de alguns deles se apresentam desesperançosos em relação às tentativas de interromper tal comportamento.

O DSM-5 exige que os critérios clínicos significativos a serem preenchidos devem incluir: o comportamento de arrancar os cabelos pode causar estresse clinicamente significativoou prejuízo no funcionamento social, ocupacional, ou em outras áreas (American Psychiatric Association -APA,2013). Os indivíduos com tricotilomania podem sentir vergonha extrema, embaraço, ou perda do controle no âmbito da incapacidade de interromper o comportamento de arrancar os cabelos. Eles podem despender uma boa quantidade de tempo camuflando a sua perda de cabelos, evitarem uma variedade de situações sociais, ou evitarem, em última instância, os relacionamentos, com o intuito de assegurar que a perda de cabelo não é visualizada pelos outros (WOODS; FLESSNER; FRANKLIN, 2006). De um modo geral, nem sempre os amigos mais chegados e os familiares estão conscientizados acerca da dimensão do comportamento de arrancar os cabelos e do sentimento de vergonha.

O comportamento de arrancar os cabelos ou a perda dos cabelos não pode ser atribuível a outra condição médica e não deve ser mais bem explicada por outro transtorno psiquiátrico. Várias condições dermatológicas são caracterizadas pela perda dos cabelos, e o comportamento de arrancar os cabelos pode ser visto em um número de outras condições psiquiátricas. Então, os indivíduos com tricotilomania podem arrancar os cabelos na tentativa de alterar um defeito na aparência. De um modo menos comum, o comportamento de arrancar os cabelos pode ocorrer como resposta a uma alucinação relativa a um transtorno psicótico.

\section{Etiologia}

\section{Teorias Genéticas}

Estudos que envolvem gêmeos e famílias fornecem evidências preliminares de que a tricotilomania é detentora de um componente genético significativo, e que também é mais comum em parentes de indivíduos com TOC (NOVAK; KEUTHEN; STEWART, 2009). 
Há também uma evidência limitada de que as variantes em certos genes candidatos (incluindo os genes localizados no sistema neurotransmissor serotoninérgico, e um gene que codifica uma proteína denominada por SAPAP3, que é encontrada nas sinapses de excitação, responsivas ao glutamato com expressão particularmente elevada no estriado) estão associadas à tricotilomania, e ainda pode desempenhar um papel contributivo na patogênese (HEMMINGS; KINNEAR; LOCHNER, 2006, BOARDMANN; VAN DER MERWE; LOCHNER, 2011).

\section{Teorias Neurológicas}

Tendo como base um nível cognitivo-afetivo, existem algumas evidências de que os indivíduos com tricotilomania demonstram problemas na inibição motora, apontando para um envolvimento do circuito corticostriado (Chamberlain, Blackwell, \& Fineberg, 2006). A partir de uma perspectiva evolucuonária, a tricotilomania pode ser conceitualizada em termos de preparação distorcida, o que implica potencialmente essas estruturas. Estudos por neuroimagem fornecem algum suporte para esta hipótese, com um número de estudos que se deparam com anormalidades nos volumes corticostriados ou integridade do trato (CHAMBERLAIN; MENZIES; FINEBERG, 2008, ROOS; FOUCHE; STEIN, 2013). Os modelos animais sugerem que determinados componentes moleculares do referido circuito pode desempenhar um papel na patogênese da tricotilomania (GREER; CAPECCHI, 2002, WELCH; LU; RODRIGUIZ, 2007).

\section{Teorias Psicológicas}

Os modelos cognitivo-comportamentais sugerem que o comportamento de arrancar os cabelos pode servir para modular os níveis de excitação. Tanto o reforço positivo (por exemplo, prazer proporcionado pelo comportamento de puxar os cabelos) quanto o reforço negativo (por exemplo, a redução da ansiedade) têm sido hipóteses no âmbito da contribuição da manutenção do comportamento de arrancar os cabelos. 


\section{Referências}

ADLER, C. M; MCDONOUGH-RYAN, P.; SAX, K. W; HOLLAND, S. K; ARNDT, $S$; STRAKOWSKI, S. M. fMRI of neuronal activation with symptom provocation in unmedicated patients withobsessive compulsive disorder. Journal of Psychiatric Research, v. 34, n. 4-5, p. 317-324, 2000.

ALBERTINI, R. S; PHILLIPS, K. A. Thirty-three cases of body dysmorphic disorder in children and adolescents. Journal of the American Academy of Child andAdolescent Psychiatry, v. 38, n.4, p. 453-459, 1999

ALONSO, P; GRATACOS, M; MENCHON, J. Genetic susceptibility to obsessivecompulsive hoarding: the contribution of neurotrophic tyrosine kinase receptor type 3 gene. Genes, Brain, and Behavior, v.7, n.7, p. 778-785, 2008.

ALTAMURA, C; PALUELLO, M. M; MUNDO, E., MEDDA, S; MANNU, P. Clinical and subclinical body dysmorphic disorder. European Archives of Psychiatry and ClinicalNeuroscience, v. 251, n.3, p.105-108, 2001.

AMERICAN PSYCHIATRIC ASSOCIATION - APA Diagnostic and Statistical Manual of Mental Disorders, 5th edn. (DSM-5). Arlington, VA: American Psychiatric Publishing, 2013.

BAER, L; MINICHIELLO,W.E Behavior therapy for obsessive-compulsive disorder. In: JENIKE, MA; BAER, L; MINICHIELLO, WE (Orgs.) Obsessive-Compulsive Disorders: Theory and Management. St Louis, MO: Year Book Medical Publishers. 1990.

BAXTER, L.R. Neuroimaging studies of obsessive compulsive disorder. Psychiatric Clinics of North America, v.15, n.4, , p. 871-884, 1992.

BAXTER, L.R; SCHWARTZ, J.M; GUZE, B.H. Brain imaging: Toward a neuroanatomy of OCD. In: ZOHAR, J; INSEL, T; RASMUSSEN, S. (Orgs.). The psychobiology of obsessive-compulsive disorder. New York: Springer, 1991.

BERTHIER, M. L; KULISEVSKY, J; GIRONELL, A; LÓPEZ, O. L. Obsessive compulsive disorder and traumatic brain injury: Behavioral, cognitive, and neuroimaging findings. Neuropsychiatry, Neuropsychology, E Behavioral Neurology, v.14, p.23-31, 2001

BIENVENU, O.J., SAMUELS, J.F., WUYEK, L.A. Is obsessive-compulsive disorder an anxiety disorder, and what, if any, are spetrum conditions? A family study perspective. Psychological Medicine, v.13, p. 1-13, 2011.

BJORNSSON, A. S., DYCK, I., MOITRA, E., STOUT, R. L.,WEISBERG, R. B., KELLER, M. B., PHILLIPS, K. A. The clinical course of body dysmorphic disorder in the Harvard/Brown Anxiety Research Project (HARP). Journal of Nervous and Mental Disease, v. 199, n. 1, p. 55-57, 2011 
BLACK, D.W; NOYES, R; GOLDSTEIN, R.B; BLUM, N. A family study of obsessive compulsive disorder. Archives of General Psychiatry, v. 49, p.362-368, 1992.

BLOCH, M. H.; LANDEROS-WEISENBERGER, A.; SEN, S.; DOMBROWSKI, P.; KELMENDI, B.; CORIC, V.; LECKMAN, J. F. Association of the serotonin transporter polymorphism and obsessive-compulsive disorder: Systematic review. Am. J. of Med. Genet., v. 147b, n.6, p.850-858, 2008.

BOARDMAN, L., VAN DER MERWE, L., LOCHNER, C. Investigating SAPAP3 variants in the etiology of obsessive-compulsive disorder and trichotillomania in the South African white population. Comprehensive Psychiatry, v.52, p. 181-187, 2011.

BOHNE, A., KEUTHERN, N. J., WILHELM, S., DECKERSBACK, T., JENIKE, M.A. (2002). Prevalence of symptoms of body dysmorphic disorder and its correlates: A cross-cultural comparison. Psychossomatics, v.43, p.486-490.

BREITER, H.C., RAUCH, S.L., KWONG, K.K., BAKER, J.R., WEISSKOFF, R.M., KENNEDY, D.N. Functional magneticresonance imaging of symptom provocationin obsessive-compulsive disorder. Archives of General Psychiatry, v.53, p.595-606, 1996 BUCHANAN, B.G., ROSSELL, S.L., MALLER, J.J. Brain connectivity in body dysmorphic disorder compared with controls: a diffusion tensor imaging study. Psychological Medicine, v.43, n. 12, p. 2513-2521, 2013.

BUHLMANN, U., ETCOFF, N.L., WILHELM, S. Emotion recognition bias for contempt and anger in body dysmorphic disorder. Journal of Psychiatric Research, v.40, p. 105-111, 2006.

CHAMBERLAIN, S.R., BLACKWELL, A.D., FINEBERG, N.A. Strategy implementation in obsessive-compulsive disorder and trichotillomania. Psychological Medicine, v. 36, p. 91-97, 2006.

CHAMBERLAIN, S.R., MENZIES, L.A., FINEBERG, N.A. Grey matter abnormalities in trichotillomania: morphometric magnetic resonance imaging study. British Journal od Psychiatry, v. 193, p. 216-221, 2008.

CHOW, T.W., FAM, D., GRAFF-GUERRERO, A. Fluorodeoxyglucose positron emission tomography in semantic dementia after 6 months of memantine: an open-label pilot study. International Journal of GeriatricPsychiatry, v.28, n.3, p. 319-325, 2012.

CLARK, D. A.; O'CONNOR, K. Thinking is believing: Ego-dystonic intrusivethoughts in obsessive-compulsive disorder. In: Clark, D. A (Org.), Intrusive thoughts inclinical disorders. New York, NY: Guilford, 2005, p. 145-174.

DE BRUIJN, E.R., HULSTIJN, W., VERKES, R.J. Drug-induced stimulation and suppression of action monitoring in healthy volunteers. Psychopharmacology, $v$. 177, n.1-2, p.151-160, 2004. 
DE HAES, J.I., MAGUIRE RP, JAGER, P.L. Methylphenidate-induced activation of the anterior cingulate but not the striatum: a PET study in healthy volunteers. Human Brain Mapping, v.28, n.7, p. 625-635, 2007.

DIDIE, E. R., MENARD, W., STERN, A. P., PHILLIPS, K. A. Occupational functioning and impairment in adults with body dysmorphic disorder. Comprehensive Psychiatry, v. 49, n.6, p.561-559, 2008.

DUNAI, J., LABUSCHAGNE, I., CASTLE, D.J. Executive function in body dysmorphic disorder. Psychological Medicine, v.40, p.1541-1548, 2010.

FEUSNER, J. D., ARIENZO, D., LI, W. White matter microstruture in body dysmorphic disorder and its clinical correlates. Psychological Medicine, v.41, p.132-140, 2013.

FEUSNER, J. D., HEMBACHER, E., MOLLER, HAbnormalities of object visual processing in body dysmorphic disorder. Psychological Medicine. v.41, p.2385-2397, 2011.

FEUSNER, J. D., HEMBACHER, E., PHILliPS, K.A. The mouse who couldn't stop washing: pathologic grooming in animals and humans. CNS Spectrums, v.14, p.503-513, 2009.

FEUSNER, J. D; MOODY, T; HEMBACHER, E. Abnormalities of visual processing and frontostriatal systems in body dysmorphic disorder. Archives of General Psychiatry, v.67, p. 197-205, 2010.

FREUD, S. An infantile neurosis.[1917-1919] The Standard Edition of the Complete Psychologic Works of Sigmund Freud, Vol. XVII. London: Hogarth Press, 1963.

FROST, R; TOLIN, D; STEKETEE, G; FITCH, K; SELBO-BRUNS, A. Excessive acquisition in hoarding. Journal of Anxiety Disorders, v. 23, n. 5, p.632-639, 2009.

GARNER, J.P., THOGERSON, C.M., DUFOUR, B.D. Reverse-translational biomarker validation of abnormal repetitive behaviors in mice: an illustration of the $4 \mathrm{P}^{\prime} \mathrm{s}$ modeling approach. Behavioural Brain Research, v.219, p.189-196, 2011.

GRANT, J.E., ODLAUG, B.L., CHAMBERLAIN, S.RA. Cognitive comparison of pathological skin picking and trichotillomania. Journal of Psychiatric Research, v.45, p. 1634-1638, 2011.

GREER, J.M., CAPECCHI, M.R. Hoxb8 is required for normal grooming behavior in mice. Neuron, v. 33, n. 1, p.23-34, 2002.

GRISHAM, J. R; FROST, R. O; STEKETEE, G; KIM, H. J; HOOD, S. Age of onset of compulsive hoarding. Journal of Anxiety Disorders, v. 20, n.5, p.675-686, 2006.

GUSTAD, J., PHILLIPS, K.A. Axis I comorbidity in body dysmorphic disorder. Comprehensive Psychiatry, v.44, n.4, p. 270-276, 2003. 
HEMMINGS, S.M.J., KINNEAR, C.J., LOCHNER, C. Genetic correlates in trichotillomania - a case-control association study in the South African Caucasian population. Israel Journal of Psychiatry and Related Sciences, v. 43, n.2, p.93-101, 2006.

HIRSCHTRITT, B.A., MATHEWS C.A. Genetics and family models of hoarding disorder. In: FROST R.O.; STEKETEE, G (Orgs.) Oxford Handbook ofHoarding and Acquiring. Oxford: Oxford University Press, p. 159-176, 2014.

HOLLANDER, E., LIEBOWITZ, M.R., ROSEN, W.G. Neuropsychiatric and neuropsychological studies in obsessive compulsive disorder. In: ZOHAR, J., INSEL, T, RASMUSSEN, S (Orgs.), The psychobiology of obsessive-compulsive disorder. ( $\mathrm{p}$. 126-145). New York: Springer, 1991.

HWANG, J. P; TSAI, S.J; YANG, C.H; LIU, K.M; LIRNG, J.F. Hoarding behavior in dementia. A preliminary report. American Journal of Geriatric Psychiatry: Official Journal of the American Association for Geriatric Psychiatry, v.6, n.4, p.285-289, 1998.

IERVOLINO, A. C; RIJSDIJK, F. V; CHERKAS, L; FULLANA, M. A; MATAIX-COLS, D. A multivariate twin study of obsessive-compulsive symptom dimensions. Archives of General Psychiatry, v. 68, n.6, p. 637-644, 2011.

INSEL, T. R. Toward a neuroanatomy of obsessive-compulsive disorder. Archives of General Psychiatry, v. 49, n.9, p.739-744, 1992.

JACOBI, F; WITTCHEN, H. U; HOLTING, C.; HOFLER, M; PFISTER, H; MULLER, N; LIEB, F. Prevalence, co-morbidity and correlates of mental disorders in the general population: Results from the German Health Interview and Examination Survey. Psychological Medicine, v.34, n.4, p.597-611, 2004

JENIKE, M. A. Obsessive-compulsive disorder: A question of a neurologic lesion. Comprehensive Psychiatry, v.25, p.298-304, 1984.

JOO, E.Y; TAE, W.S; JUNG, K.Y. Cerebral blood flow changes in man by wake-promoting drug, modafinil: a randomized double blind study. Journal of Sleep Research, v.17, n.1, p.82-88, 2008.

KIM, J.J; LEE, M. C; KIM, J; KIM, I. Y; KIM, S. I; HAN, M. H. Grey matter abnormalities in obsessive compulsive disorder: Statistical parametric mapping of segmented magnetic resonance images. British Journal of Psychiatry, oct., v.179, p.330-334, 2001.

KIM, H.J; STEKETEE, G; FROST, R.O. Hoarding by elderly people. Health and Social Work, v. 26, n.3, p.176-184, 2001.

LOCHNER, C; GRANT., J.E; ODLAUG, B.L. DSM-5 field survey: skin picking disorder. Annals of Clinical Psychiatrym, v. 24, p. 300-304, 2012. 
LOCHNER, C; GRANT, J.E; ODLAUG, B.L. DSM-5 field survey: hair-pulling disorder (trichotillomania). Depression and Anxiety, v.29, n. 12, p.1025-1031, 2012.

LOCHNER, C; KINNEAR, C.J; HEMMINGS, S.M. Hoarding in obsessive-compulsive disorder: clinical and genetic correlates. Journal of Clinical Psychiatry, v.66, n.9, p.1155-1160, 2005.

MACKINNON, R.A; MICHELS, R. The obsessive patient. In: MACKINNON, RA; MICHELS, R (Orgs.) The Psychiatric Interview in Clinical Practice. Philadelphia, PA: WB Saunders, pp. 89-109, 1971.

MATAIX-COLS, D; FROST, R.O; PERTUSA, A; CLARK, L.A; SAXENA, S; LECKMAN, J.F; WILHELM, S. Hoarding disorder: A new diagnosis for DSM-V? Depression and Anxiety, v. 27, n.6, p.556-572, 2010

MATAIX-COLS, D; WOODERSON, S; LAWRENCE, N. Distinct neural correlates of washing, checking, and hoarding symptom dimensions in obsessive-compulsive disorder. Archives of General Psychiatry, v.61, n.6, p.564-576, 2004

MICALLEF, J; BLIN, O. Neurobiology and clinical pharmacology of obsessive-compulsive disorder. Clinical Neuropharmacology, v.24, p. 191-207, 2001.

MODELL, J. G; MOUNTZ, J. M; CURTIS, G. C; GREDEN, J. F. Neurophysiologic dysfunction in basal ganglia/limbic striatal and thalamocortical circuits as a pathogenetic mechanism of obsessive-compulsive disorder. Journal of Neuropsychiatry $\mathcal{E}$ Clinical Neurosciences, v.1, n.1, p. 27-36, 1989.

MONZANI, B; RIJSDIJK, F; CHERKAS, L. Prevalence and heritability of skin picking disorder in an adult community sample: a twin study. American Journal of Medical Genetics. Part B, Neuropsychiatric Genetics, 159b, n.5, p. 605-610, 2012.

MONZANI, B; RIJSDIJK, F; IERVOLINO, A.C. Evidence for a genetic overlap between body dysmorphic disorder concerns and obsessive-compulsive symptoms in an adult female community twin sample. American Journal of Medical Genetics Part B, 159B, p.376-282, 2012.

MUINOS-GIMENO, M; GUIDI, M; KAGERBAUER, B. Allele variants in functional MicroRNA target sites of the neurotrophin-3 receptor gene (NTRK3) as susceptibility factors for anxiety disorders. Human Mutation,v.30, n.7, p.1062-1071, 2009.

NAHAS, Z; GEORGE, M.S; HORNER, M.D. Augmenting atypical antipsychotics with a cognitive enhancer (donepezil) improves regional brain activity in schizophrenia patients: a pilot double-blind placebo controlled fMRI study. Neurocase, v.9, n.3, p. 274-282, 2003. 
NOVAK, C.E., KEUTHEN, N.J., STEWART, S.E. A twin concordance study of trichotillomania. American Journal of Medical Genetics. Part B, Neuropsychiatric Genetics, oct 5, v.150b, n.7, p. 944-949, 2009.

PATRONEK, G.J; NATHANSON, J.N. A theoretical perspective to inform assessment and treatment strategies for animal hoarders. Clinical Psychology Review, v. 29, n.3, p. 274-281, 2009.

PAULS, D.L; ALSOBROOK, J.P; GOODMAN, W; RASMUSSEN, S; LECKMAN, J.F..A family study of obsessive-compulsive disorder. American Journal of Psychiatry, v. 152, p. 76-84, 1995

PAULS, D.L; RAYMOND,C.L; ROBERTSON, M. The genetics of obsessive compulsive disorder: A review. In: J. ZOHAR,T; INSEL, S. RASMUSSEn (Orgs.). The psychobiology of obsessive-compulsive disorder (p. 89-100). New York: Springer. 1991.

PERTUSA, A; FROST, R.O; FULLANA, M.A. Rfining the diagnostic boundaries of compulsive hoarding: a criticale review. Clinical Psychology Review, v.30, n.4, p.371-386, 2010.

PERUGI, G; AKISKAL, H.S; GIANOTTI, D; FRARE, F; DI VAIO, S; CASSANO, GB. Gender-related differences in body dysmorphic disorder. Journal of Nervous and Mental Disease, set. v. 185, n. 9, p.578-582, 1997.

PHILLIPS, K.A. The broken mirror: Understanding and treating body dysmorphic disorder. New York: Oxford University Press, 2005.

PHILLIPS, K.A "I look like a monster": Pharmacotheraphy and cognitive-behavioral therapy for body dysmorphic disorder. In: . Spitzer, R. L; First, M. B; Williams, J. B. W; Gibbon, M. (Orgs.), DSM-IV-TR case book (Vol. 2) experts tell how they treated their own patients (pp. 263-276). Washington, DC: American Psychiatric Publishing, 2006.

PHILLIPS, K.A. Understanding Body Dysmorphic Disorder: An Essential Guide. New York,NY: Oxford University Press, 2009.

PHILLIPS, K.A; MCELROY, S.L; DWIGHT, M.M; EISEN, J.L; RASMUSSEN, S.A. Delusionality and response to open-label fluvoxamine in body dysmorphic disorder. Journal of Clinical Psychiatry, v.62, p. 87-91, 2001.

PHILLIPS, K.A; WILHELM, S; KORAN, L.M. Body dysmorphic disorder: some key issues for DSM-V. Depression and Anxietym v.27, p.573-591, 2010.

PHILLIPS, K.A; WILHELM, S; KORAN, L.M; DIDIE, E.R; FALLON, B.A; FEUSNER, J; STERN, D.J. Body dysmorphic disorder. Some key issues for DSM-V. Depression and Anxiey, v.27, n. 6, p.573-591, 2010 
PIGOTT, T, A; MYERS, K. R; WILLIAMS, D. A. Obsessive-compulsive disorder: A neuropsychiatric perspective. In: Rapee, R.M. (Org.), Current controversies in theanxiety disorders. (pp. 13-160). New York:Guilford Press, 1996.

RACHMAN, S; ELLIOT, C.M; SHAFRAN, R. Separating hoarding from OCD. Behaviour Research and Therapy, v.47, n.6, p.520-522, 2009.

RACHMAN, S.J; HODGSON, R.J. Obsessions and Compulsions. Englewood Cliffs, NJ: Prentice-Hall, 1980.

RAPOPORT, J.L. Recent advances in obsessive-compulsive disorder. Neuropsychopharmacology, v. 5, n. 1, p. 1-10, 1991.

RAPOPORT, J.L; SWEDO, S.E; LEONARD, H.L. Childhood obsessive compulsive disorder. Journal of Clinical Psychiatry, v.53 (Suppl-4), p. 11-16, 1992.

RAUCH, S. L; DOUGHERTY, D.D; COSGROVE, G. R; CASSEM, E.H; ALPERT, N.M; PRICE, B.H; NIERENBERG, A.A; MAYBERG, H.S; BAER,L; JENIKE, M.A; FISCHMAN, A. J. Cerebral metabolic correlates as potential predictors of response to anteriorcingulotomy for obsessive compulsive disorder. Biological Psychiatry, nov. v.50, n.9, p. 659-667, 2001.

RAUCH, S.L; JENIKE, M.A; ALPERT, N.M; BAER, L; BREITER, H.C; SAVAGE, C. R. Regional cerebral blood flow measured during symptom provocation in obsessive-compulsive disorder using oxygen 15-labeled carbon dioxide and positron emission tomography. Archives of General Psychiatry, v.51, n.1, p. 62-70, 1994.

RIEF, W; BUHLMANN, U; WILHELM, S; BORKENHAGEN, A; BRAHLER, E. The prevalence of body dysmorphic disorder. A population-based survey. Psychological Medicine, v.36, n. 6, p.877-885, 2006.

ROOS, A; FOUCHE, J.P; STEIN, D.J. White matter integrity in hairpulling disorder (trichotillomania). Psychiatry Research, v.211, n.3, p. 246-250, 2013.

RUSCIO, A.M; STEIN, D.J; CHIU, W.T; KESSLER, R.C. The epidemiology of obsessive-compulsive disorder in the National Comorbidity Survey Replication. Molecular Psychiatry, v. 15, n.1, p.53-63, 2010.

SAMUELS, J; BIENVENU, O; PINTO, A; FYER, A; MCCRACKEN, J; RAUCH, S; KNOWLES, J. Hoarding in obsessivecompulsive disorder: Results from the OCD Collaborative Genetics Study. Behaviour Research and Therapy, v.45, n.4, p.673-686. 2007.

SAMUELS, J.F; BIENVENU, O.J; PINTO, A. Hoarding in obsessive-compulsive disorder: results from the OCD Collaborative Genetics Study. Behaviour Research and Therapy, v. 45, n.4, p. 673-686, 2007. 
SAMUELS, J.F; SHUGART, Y.Y; GRADOS, M.A. Significant linkage to compulsive hoarding on chromosome 14 in families with obsessive-compulsive disorder: results from the OCD Collaborative Genetics Study. American Journal of Psychiatry, v.164, n.3, p.493-499, 2007.

SAXENA, S; BRODY, A.L; SCHWARTZ, J.M; BAXTER, L.R. Neuroimaging and frontal-subcortical circuitry in obsessive compulsive disorder. British Journal of Psychiatry, suppl, v. 35, 26-37, 1998.

SAXENA, S; RAUCH, S. L. Functional neuroimaging and the neuroanatomy of obsessive-compulsive disorder. Psychiatric Clinics of North America, v. 23, n. 3, p.563-586, 2000.

SEEDAT, S; MATSUNAGA, H. Cross-national and ethnic issues in OC spectrum disorders. CNS Spectrum, v.12, p.392-400, 2006.

SKOOG, G., SKOOG, I. A 40-year follow-up of patients with obsessive-compulsive disorder. Archives of General Psychiatry, v. 56, p. 121-130, 1999.

SLYNE, K; TOLIN, D.F. The neurobiology of hoarding disorder. In: FROST, RO; STEKETEE, G (Orgs.) Oxford Handbook of Hoarding and Acquiring. Oxford: Oxford University Press, pp. 177-186, 2014.

SMOLLER, J.W; FINN, C; WHITE, C. The genetics of anxiety disorders: An overview. Psychiatric Annals, v.30, p.745-753, 2000.

SPENCE, S.A; GREEN, R.D; WILKINSON, I.D. Modafinil modulates anterior cingulate function in chronic schizophrenia. British Journal of Psychiatry, jul. v.187, p. 55-61, 2005.

STEKETEE, G., FROST, R. Compulsive hoarding: Current status of the research. Clinical Psychology Review, v. 23, p. 905-927, 2003.

STERN, R.S.; COBB, J.P. Phenomenology of obsessive- compulsive neurosis. British Journal of Psychiatry, v. 132, n. 3, p. 233-239, 1978.

SUZUKI, K; TAKEI, N; KAWAI, M; MINABE, Y; MORI, N. Is taijin kyofusho a culture-bound syndrome? American Journal of Psychiatry, v.160, n.7, p.1358, 2003.

SWEDO, S. E; LEONARD, H.L; GARVEY, M; MITTLEMAN, B; ALLEN, A.J; PERLMUTTER, S. Pediatric autoimmune neuropsychiatric disorders associated with streptococcal infections: Clinical description of the fi rst 50 cases. American Journal of Psychiatry, v.155, p.264-271, 1998.

TOLIN, D. F; FROST, R. O; STEKETEE, G; GRAY, K. D; FITCH, K. E. The economic and social burden of compulsive hoarding. Psychiatry Research, v. 160, n.2, p. 200-211, 2008. 
TOLIN, D.F; KIEHL, K.A; WORHUNSKY, P. An exploratory study of the neural mechanisms of decision making in compulsive hoarding. Psychological Medicine, v.39, n.2, p.325-336, 2009.

TORGERSEN, S. G. Genetic factors in anxiety disorders. Archives of General Psychiatry, v. 40, p. 1085-1089, 1983.

TORRES, A. R; PRINCE, M. J; BEBBINGTON, P. E; BHURGA, D; BRUGHA, T. S; FARRELL, M; SINGLETON, N. Obsessive-compulsive disorder: Prevalence, comorbidity, impact, and help-seeking in the British National Psychiatric Morbidity Survey of 2000. American Journal of Psychiatry, v. 163, p.1978-1985, 2006.

TUCKER, B.T.P; WOODS, D.W; FLESSNER, C.A. The Skin Picking Impact Project: phenomenology, interference, and treatment utilization of pathological skin picking in a population-based sample. Journal of Anxiety Disorders, v.25, p.88-95, 2011.

VEALE, D. Outcome of cosmetic surgey and "DIY" surgery in patients with body dysmorphic disorder. Psychiatric Bulletin, v.24, p. 218-221, 2000.

WELCH, J.M; LU, J; RODRIGUIZ, R.M., et. al. Cortico-striatal synaptic defects and OCD-like behaviours in Sapap3-mutant mice. Nature, v.448, n.7156, p. 894-900, 2007.

WENDLAND, J.R; MOYA, P.R; TIMPANO, K.R. A haplotype containing quantitative trait loci for SLC1A1 gene expression and its association with obsessive-compulsive disorder. Archives of General Psychiatry, v.66, n.4, p.408-416, 2009.

WHEATON, M.G., ABRAMOWITZ, J.S., FABRICANT, L.E, et. al. Is hoarding a symptom of obsessive compulsive disorder? International Journal of Cognitive Therapy, v. 4, n.3, 225-238, 2011.

WOODS, D., FLESSNER, C.A., FRANKLIN, M.E, et. al. The Trichotillomania Impact Project (TIP): exploring phenomenology, functional impairment, and treatment utilization. Journal of Clinical Psychiatry, v. 67, n. 12, p.1877-1888, 2006.

ZHANG, H; LECKMAN, J.F; PAULS, D.L. Genomewide scan of hoarding in sib pairs in which both sibs have Gilles de la Tourette syndrome. American Journal of Human Genetics, v.70, n.4, p.896-904, 2002.

Contato do Autor:

Fernando Filipe Paulos Vieira - email: fernandofpvieira@gmail.com

Recebido em 10/05/2017

Aceito em 15/10/2017 